July 8, 2021

\title{
Testing numerical evolution with the shifted gauge wave
}

\author{
Maria C. Babiuc ${ }^{1}$, Béla Szilágyi ${ }^{2}$, Jeffrey Winicour ${ }^{1,2}$ \\ 1 Department of Physics and Astronomy \\ University of Pittsburgh, Pittsburgh, PA 15260, USA \\ 2 Max-Planck-Institut für Gravitationsphysik, \\ Albert-Einstein-Institut, \\ 14476 Golm, Germany
}

\begin{abstract}
Computational methods are essential to provide waveforms from coalescing black holes, which are expected to produce strong signals for the gravitational wave observatories being developed. Although partial simulations of the coalescence have been reported, scientifically useful waveforms have so far not been delivered. The goal of the AppleswithApples (AwA) Alliance is to design, coordinate and document standardized code tests for comparing numerical relativity codes. The first round of AwA tests have now being completed and the results are being analyzed. These initial tests are based upon periodic boundary conditions designed to isolate performance of the main evolution code. Here we describe and carry out an additional test with periodic boundary conditions which deals with an essential feature of the black hole excision problem, namely a nonvanishing shift. The test is a shifted version of the existing AwA gauge wave test. We show how a shift introduces an exponentially growing instability which violates the constraints of a standard harmonic formulation of Einstein's equations. We analyze the Cauchy problem in a harmonic gauge and discuss particular options for suppressing instabilities in the gauge wave tests. We implement these techniques in a finite difference evolution algorithm and present test results. Although our application here is limited to a model problem, the techniques should benefit the simulation of black holes using harmonic evolution codes.
\end{abstract}

PACS numbers: PACS number(s): 04.20Ex, 04.25Dm, 04.25Nx, 04.70Bw

\section{INTRODUCTION}

Computational methods are essential to provide the waveform from the coalescence of black holes, which is expected to produce a strong signal for the gravitational wave observatories being developed. The importance of the binary black hole problem to the success of LIGO and LISA has led to major computational efforts, most notably the Binary Black Hole Grand Challenge. Although this Grand Challenge had intermediate successes [1, 2, 3], scientifically useful waveforms were not delivered. At present, this remains a problem beyond the reach of any existing code.

A recent study [4] of large scale scientific code projects at the Livermore, Los Alamos and Sandia National Laboratories, funded under the U.S. Department of Energy's Accelerated Strategic Computing Initiative (ASCI), identified three necessary elements for success: verification, validation and quality management. In the absence of any of those three requirements, the report concluded that the results would have little scientific impact because of the impossibility to judge code reliability. Although the ASCI projects involved highly experienced and qualified teams at laboratories with ample resources, only one third of the projects succeeded as planned, another third succeeded later than planned and the remaining were eventually abandoned. The failed projects had overly ambitious schedules and goals and lacked a conservative methodology that minimized risk. It was expected that the failure rate would have been much higher for simulation projects without the experience and resources of the ASCI teams.

The lessons learned from the ASCI and other studies have been reviewed by Post and Votta [5]. Their experience with the peer review process in computational science was that it is not as effective as in experiment or theory, because the many possible sources of hidden defects make the referee rely too heavily on plausibility checks as opposed to independent reproduction of results. Some of their observations, which are presented below in quotes, are very pertinent to numerical relativity: "New methods of verifying and validating complex codes are mandatory if computational science is to fulfill its promise for science and society". The validation of a code implies that the predictions of the code are in accord with observed phenomena. For the present status of the binary black hole problem, in the absence of any empirical observations, the burden falls completely on verification.

Post and Votta list five verification techniques, each one having limited effectiveness by itself:

1. "Comparing code results with an exact answer".

2. "Establishing that the convergence rate of the truncation error with changing grid spacing is consistent with expectations". 
3. "Comparing calculated with expected results for a problem especially manufactured to test the code".

4. "Monitoring conserved quantities and parameters, preservation of symmetry properties and other easily predictable outcomes".

5. "Benchmarking - that is, comparing results from those with existing codes that can calculate similar problems".

The importance of the first four techniques has been recognized by most numerical relativity groups and their implementation in practice has improved the integrity of the field. Individual groups cannot easily carry out the fifth technique independently. This was the motivation behind formation of the AppleswithApples (AwA) Alliance, whose goal is to design, coordinate and document standardized code tests for comparing numerical relativity codes. A pivotal step has been the documentation of a first round of AwA tests based upon periodic boundary condition (equivalent to a 3-torus with no boundary) [6], designed to isolate performance of the main evolution code. The choice of initial AwA tests was biased by considerations of expediency arising from the state of the field at the time. The cross fertilization between computational mathematics and numerical relativity was just entering a productive stage. Only a few groups had based their codes upon well-posed symmetric or strongly hyperbolic formulations of Einstein's equations and fewer groups had an understanding of how to treat boundary conditions. Detailed specifications of a second round of tests, involving boundaries (essential for the black hole excision problem), have now been proposed [7].

Post and Votta emphasized that

- "Verification and validation establish the credibility of code predictions. Therefore it's very important to have a written record of verification and validation results."

- "Without such programs, computational science will never be credible."

The first round of AwA tests have now been completed using codes based upon most of the prominent numerical relativity formalisms. Results carried out by the participating groups can be viewed in the Alliance CVS depository. Instructions for accessing the data in the CVS are available at the Alliance website 7]. A second paper is in preparation which discusses these first round test results with respect to code performance and improvements in the test specifications [8].

Here we describe and carry out an additional test based upon periodic boundary conditions which deals with an essential feature of the black hole excision problem, namely a non-vanishing shift. The test is a shifted version of the existing AwA gauge wave test. We detail the test specifications in Sec. III

In Sec. III we describe some instabilities associated with the gauge wave and shifted gauge wave tests. A discussion of the Cauchy problem for general relativity depends upon a choice of gauge conditions which reduce Einstein's equations to hyperbolic form. See [9] for a recent review. The simplest reduction is in terms of harmonic coordinates [10, 11], for which well-posedness of the Cauchy problem was first established [12]. Previous work [13] has revealed that the gauge wave without shift has a constraint preserving instability in harmonic coordinates, i.e the gauge wave metric has exponentially growing perturbations which satisfy the harmonic conditions and Einstein's equations. We show here how a shift introduces a new type of exponentially growing instability in the standard harmonic reduction of Einstein's equations.

In Sec.IV] we summarize various options for constructing a hyperbolic evolution algorithm based upon the harmonic formulation. Our discussion centers around the standard form of the harmonic formulation adopted in most recent analytic treatments. Although the well-posedness of the Cauchy problem guarantees the existence and uniqueness of a solution with continuous dependence on the initial data, this is only a necessary condition for computational success and is not sufficient. Simulations have shown that instabilities in the gauge wave metrics can quickly introduce unacceptable error even with a convergent code [13]. In the case of the gauge wave without shift, these instabilities can be suppressed by implementing discrete conservation laws obeyed by the principle part of the evolution system [13]. However, in the case of the shifted gauge wave this technique is not effective by itself because the instability is excited by nonlinear terms. In Sec. IV] we discuss options at the analytic level for suppressing this instability in the shifted gauge wave test. One option is to adjust the nonlinear terms in the evolution system by adding terms which vanish modulo the harmonic constraints. Another option is the inclusion of harmonic gauge forcing terms, which in principle allow the simulation of any nonsingular spacetime region. Gauge forcing terms not only allow the flexibility to "steer" around coordinate pathologies that might arise but they also allow the adaptability to carry out standardized tests in any specified gauge.

Section $\nabla$ describes the implementation of the harmonic formulation as a finite difference evolution code. We base our approach on a code [14], the Abigel code which was developed to implement a well-posed, constraint preserving version of the harmonic initial-boundary value problem (IBVP). Here we confine our attention to the Cauchy problem. In future work we will extend our test results to the IBVP. Since the preliminary testing of the Abigel code, considerable improvement has been made in the underlying numerical techniques. The study of a model nonlinear scalar wave [15] 
shows that semi-discrete conservation laws and summation by parts can be used to formulate stable algorithms. In Section $\nabla$ we generalize these techniques to the reduced harmonic Einstein equations. These techniques also have potential application to the $Z 4$ system [16] which generalizes the harmonic formulation.

In Sec. VI] we calibrate the stability, convergence and performance of the code using the gauge wave and shifted gauge wave metrics. We show how numerical noise excites instabilities that can be cured by a combination of discrete conservation laws and constraint adjustments.

\section{THE SHIFTED GAUGE WAVE TEST}

The standard AwA gauge wave test is based upon the flat metric

$$
d s^{2}=(1-H)\left(-d t^{2}+d x^{2}\right)+d y^{2}+d z^{2},
$$

where

$$
H=H(x-t)=A \sin \left(\frac{2 \pi(x-t)}{d}\right)
$$

is a sinusoidal wave of amplitude $A$ propagating along the $x$-axis. In order to test 2 -dimensional features, the coordinates are rotated according to

$$
x=\frac{1}{\sqrt{2}}\left(x^{\prime}-y^{\prime}\right), \quad y=\frac{1}{\sqrt{2}}\left(x^{\prime}+y^{\prime}\right)
$$

which produces a gauge wave propagating along the diagonal with dependence

$$
\sin \left(\frac{2 \pi\left(x^{\prime}-y^{\prime}-t^{\prime} \sqrt{2}\right)}{d^{\prime}}\right), \quad \text { where } \quad d^{\prime}=d \sqrt{2}
$$

Adjusting $d$ or $d^{\prime}$ to the size of the evolution domain gives periodicity in the $x$ and $y$ directions.

The shifted gauge wave is obtained from the Minkowski metric $d s^{2}=-d \hat{t}^{2}+d \hat{x}^{2}+d \hat{y}^{2}+d \hat{z}^{2}$ by the coordinate transformation

$$
\begin{aligned}
& \hat{t}=t-\frac{A d}{4 \pi} \cos \left(\frac{2 \pi(x-t)}{d}\right), \\
& \hat{x}=x-\frac{A d}{4 \pi} \cos \left(\frac{2 \pi(x-t)}{d}\right), \\
& \hat{y}=y, \\
& \hat{z}=z
\end{aligned}
$$

where $d$ is the size of the evolution domain. This leads to the 4-metric of Kerr-Schild form

$$
d s^{2}=-d t^{2}+d x^{2}+d y^{2}+d z^{2}+H k_{\alpha} k_{\beta} d x^{\alpha} d x^{\beta}
$$

where

$$
k_{\alpha}=\partial_{\alpha}(x-t)=(-1,1,0,0)
$$

and $H$ is again given by (2.2). This metric describes a shifted gauge wave of amplitude $A$ propagating along the $x$-axis. As above, the coordinate transformation (2.3) rotates the propagation direction to the diagonal.

The shifted gauge wave test is run in both axis-aligned 1D form and diagonal 2D form. We run the test with amplitude $A=0.5$. We have found that smaller amplitudes are not as efficient for revealing problems. Larger amplitudes can trigger gauge pathologies, e.g $g^{t t} \geq 0$ (breakdown of the spacelike nature of the Cauchy hypersurfaces), more quickly and complicate code comparisons.

We specify the wavelength $d=1$ in the $1 \mathrm{D}$ simulations and $d^{\prime}=\sqrt{2}$ in the $2 \mathrm{D}$ simulations. We find that at least 50 grid points lead to reasonable simulations for more than 10 crossing times and therefore make the following choices for the computational grid:

- Simulation domain:

$$
\begin{aligned}
& \text { 1D: } \quad x \in[-0.5,+0.5], \quad y=0, \quad z=0, \quad d=1 \\
& \text { 2D: } \quad x \in[-0.5,+0.5], \quad y \in[-0.5,+0.5], \quad z=0, \quad d^{\prime}=\sqrt{2}
\end{aligned}
$$


- Grid: $x_{n}=-0.5+n d x, \quad n=0,1 \ldots 50 \rho, \quad d x=d y=d z=1 /(50 \rho), \quad \rho=(1,2,4)$

- Time step: $d t=d x / 4=0.005 / \rho$

The $1 \mathrm{D}$ evolution is carried out for $T=1000$ crossing times, i.e. $2 \times 10^{5} \rho$ time steps (or until the code crashes). The $2 \mathrm{D}$ diagonal runs are carried out for $T=100$.

Useful output data are the profiles along the $x$-axis through the center of the grid $(y=z=0)$ of $g_{t t}, g_{x t}$, and $g_{x x}$, the $\ell_{2}$ and $\ell_{\infty}$ norms of the error and the Hamiltonian and momentum constraints, or any other constraints which arise in a particular formulation. It is also important to calculate the convergence factor of the numerical error.

\section{INSTABILITY OF THE SHIFTED GAUGE WAVE}

Both the gauge wave metric (2.1) and shifted gauge wave metric (2.6) are flat vacuum solutions of the Einstein equation in harmonic coordinates $\square x^{\mu}=-\Gamma^{\mu}=0$, where

$$
\Gamma^{\mu}=g^{\alpha \beta} \Gamma_{\alpha \beta}^{\mu}=-\frac{1}{\sqrt{-g}} \partial_{\alpha} \gamma^{\alpha \mu}
$$

and $\gamma^{\mu \nu}=\sqrt{-g} g^{\mu \nu}$.

Simulation of the AwA gauge wave without shift (2.1) is complicated by the related metric [13]

$$
d s_{\lambda}^{2}=e^{\lambda t}(1-H)\left(-d t^{2}+d x^{2}\right)+d y^{2}+d z^{2}
$$

which, for any value of $\lambda$, is a flat metric which obeys the harmonic coordinate conditions and thus represents a harmonic gauge instability of Minkowski space with periodic boundary conditions.

The shifted gauge wave (2.6) has an analogous instability

$$
d s_{\lambda}^{2}=e^{\lambda t}\left(-d t^{2}+d x^{2}\right)+d y^{2}+d z^{2}+H k_{\alpha} k_{\beta} d x^{\alpha} d x^{\beta},
$$

which is again a vacuum metric. However, the metric (3.3) is not in harmonic coordinates and has a harmonic driving term [1] $\Gamma^{\alpha}=\hat{\Gamma}^{\alpha}$, where

$$
\hat{\Gamma}^{\alpha}=-\lambda H k^{\alpha} .
$$

Thus this particular instability would only be excited in a gauge satisfying (3.4). In the standard $3+1$ description $x^{\mu}=\left(t, x^{i}\right)$, this determines the propagation of the lapse $\alpha=1 / \sqrt{-g^{t t}}$ and shift $\beta^{i}=-g^{i t} / g^{t t}$ according to

$$
\frac{1}{\alpha \sqrt{h}}\left(\partial_{t}\left(\frac{\sqrt{h}}{\alpha}\right)-\partial_{j}\left(\frac{\sqrt{h} \beta^{j}}{\alpha}\right)\right)=\hat{\Gamma}^{t}
$$

and

$$
-\frac{1}{\alpha \sqrt{h}}\left(\partial_{t}\left(\frac{\sqrt{h} \beta^{i}}{\alpha}\right)+\partial_{j}\left(\alpha \sqrt{h}\left(h^{i j}-\frac{\beta^{i} \beta^{j}}{\alpha^{2}}\right)\right)\right)=\hat{\Gamma}^{i},
$$

where $h_{i j}$ is the 3 -metric and $h=\operatorname{det}\left(h_{i j}\right)$.

The exponentially growing metrics (3.2) and (3.3) both satisfy the Einstein equations. However, the shifted gauge wave also has a different type of instability when the evolution system is taken to be the standard harmonic reduction of the Einstein tensor [9, 18]

$$
E^{\mu \nu}:=G^{\mu \nu}-\nabla^{(\mu} \Gamma^{\nu)}+\frac{1}{2} g^{\mu \nu} \nabla_{\alpha} \Gamma^{\alpha},
$$

which leads to the hyperbolic evolution equation

$$
E^{\mu \nu}=0 .
$$

(Here $\Gamma^{\nu}$ is treated formally as a vector in constructing the "covariant" derivative $\nabla^{\mu} \Gamma^{\nu}$ ). In order to see the origin of this instability first consider the spatially homogeneous case with amplitude $A=0$, for which the shifted gauge wave metric reduces to the Minkowski metric $\eta_{\mu \nu}$. Nonlinear perturbations of this metric of the Kerr-Schild form

$$
g_{\mu \nu}=\eta_{\mu \nu}+F(t) k_{\mu} k_{\nu}
$$


(where $k_{\alpha}=\partial_{\alpha}(x-t)$ as before) satisfy the reduced harmonic equations (3.8) provided

$$
E^{\mu \nu}=\frac{1}{2}\left((1+F) F_{, t t}-F_{, t} F_{, t}\right) k^{\mu} k^{\nu}=0 .
$$

This has the exponential solution

$$
F=e^{\lambda t}-1
$$

The resulting metric

$$
g_{\mu \nu}=\eta_{\mu \nu}+\left(e^{\lambda t}-1\right) k_{\mu} k_{\nu}
$$

solves the reduced equations but unlike [3.3) it does not solve the Einstein equations because the harmonic condition is not satisfied. Instead

$$
\Gamma^{\mu}=\lambda e^{\lambda t} k^{\mu}
$$

From (3.7), this implies that $G^{t \alpha}=0$, i.e. that the Hamiltonian and momentum constraints are satisfied, but that the Einstein tensor has the non-vanishing components

$$
G^{y y}=G^{z z}=-\frac{1}{2} \lambda^{2} e^{\lambda t} .
$$

Whether the unstable mode (3.12) is excited by numerical error depends upon the evolution system. A system which enforces $G^{y y}=G^{z z}=0$ would not excite this mode. In the case of harmonic evolution, the Einstein equations are satisfied only indirectly through the satisfaction of the harmonic conditions. These conditions, $\Gamma^{\mu}=0$, are the constraints of the harmonic evolution system. Because $\Gamma^{\mu}$ is not an evolution variable, error of the form (3.13) can be expected to excite the instability.

The shifted gauge wave (2.6) has an exponentially growing instability analogous to (3.12) that satisfies the reduced harmonic equations but violates the harmonic constraints. The unstable perturbation may be constructed analytically by applying the transformation (2.5) to the exponential solution (3.12) of the reduced harmonic equations. Because this transformation has the property $\hat{x}-\hat{t}=x-t$, it is simple to verify that $\Gamma^{\mu}$ transforms as a vector. As a result, the reduced equations (3.7) remain satisfied (since $G^{\mu \nu}$ is a tensor). The resulting metric is

$$
d s_{\lambda}^{2}=-d t^{2}+d x^{2}+d y^{2}+d z^{2}+\left(H-1+e^{\lambda \hat{t}}\right) k_{\alpha} k_{\beta} d x^{\alpha} d x^{\beta},
$$

where now

$$
\hat{t}=t-\frac{A d}{4 \pi} \cos \left(\frac{2 \pi(x-t)}{d}\right) .
$$

The resulting harmonic constraint violation is given by

$$
\Gamma^{\mu}=\lambda e^{\lambda \hat{t}} k^{\mu}
$$

The simulation of the shifted gauge wave by a harmonic evolution algorithm based upon the standard reduction (3.7) excites this instability, as exhibited by the results in VI However, the instability can be controlled by modifying the standard harmonic system, as discussed in the next section.

\section{THE HARMONIC CAUCHY PROBLEM}

The standard harmonic reduction of the Einstein equations is given by (3.7), with $\Gamma^{\mu}$ set to zero, which leads to a hyperbolic system (3.8) which can be cast into the flux conservative form

$$
\begin{aligned}
2 \sqrt{-g} E^{\mu \nu} & =\partial_{\alpha}\left(g^{\alpha \beta} \partial_{\beta} \gamma^{\mu \nu}\right)-2 \sqrt{-g} g^{\alpha \rho} g^{\beta \sigma} \Gamma_{\alpha \beta}^{\mu} \Gamma_{\rho \sigma}^{\nu}-\sqrt{-g}\left(\partial_{\alpha} g^{\alpha \beta}\right) \partial_{\beta} g^{\mu \nu}+\frac{1}{\sqrt{-g}} g^{\alpha \beta}\left(\partial_{\beta} g\right) \partial_{\alpha} g^{\mu \nu} \\
& +\frac{1}{2} g^{\mu \nu}\left(\frac{1}{2 g \sqrt{-g}} g^{\alpha \beta}\left(\partial_{\alpha} g\right) \partial_{\beta} g+\sqrt{-g} \Gamma_{\alpha \beta}^{\rho} \partial_{\rho} g^{\alpha \beta}+\frac{1}{\sqrt{-g}}\left(\partial_{\beta} g\right) \partial_{\alpha} g^{\alpha \beta}\right)=0 .
\end{aligned}
$$


The harmonic condition $\Gamma^{\mu}=0$ comprise the constraints which are sufficient to establish that the Einstein tensor vanishes. When the reduced harmonic equations (4.1) are satisfied, the Bianchi identities imply

$$
\nabla^{\alpha} \nabla_{\alpha} \Gamma^{\mu}+R_{\nu}^{\mu} \Gamma^{\nu}=0
$$

where the Ricci tensor satisfies

$$
R^{\mu \nu}=\nabla^{(\mu} \Gamma^{\nu)}
$$

These equations provide the key result that the harmonic conditions propagate in time if the reduced equations are satisfied. The historic proof of well-posedness of the initial value problem for Einstein's equations [12] follows from the hyperbolicity of (3.7) and (4.2). Here hyperbolicity can be defined either in terms of the second differential order systems (4.1) and (4.2), as in 12], or by reducing (4.1) and (4.2) to first order symmetric hyperbolic systems [19].

The principal part of $E^{\mu \nu}$, i.e.

$$
\partial_{\alpha}\left(g^{\alpha \beta} \partial_{\beta} \gamma^{\mu \nu}\right),
$$

has been chosen so that when the remaining nonlinear terms in $E^{\mu \nu}$ vanish (or can be neglected) the associated conservation laws suppress excitation of the vacuum solution (3.2). These conservation laws, when implemented in the discretized system, successfully suppress the instability (3.2) in the simulation of the gauge wave without shift. See 13 for details. The discussion in 13 suggests that other flux conservative forms of the principle part whose terms have an analogous conformal weight, such as

$$
\partial_{\alpha}\left(g^{\alpha \beta} \partial_{\beta} g_{\mu \nu}\right),
$$

would be equally effective at suppressing this unstable mode.

On the other hand, we have found that these conservation laws associated with the principle part (4.4) are not effective in suppressing the instability (3.15) in the simulation of the shifted gauge wave. In this case, the instability is excited by the first derivative terms terms in (4.1) which act as a nonlinear source for the principle part (4.4). This instability must be handled by a different technique. There are two straightforward generalizations of the standard harmonic treatment which leave the principle part of (4.1) unchanged, so that the well-posedness of the Cauchy problem remains intact, but modify the nonlinear terms: (i) the introduction of harmonic gauge source functions or (ii) constraint adjustment of the equations.

\section{A. Harmonic gauge source functions}

Harmonic gauge source functions $\hat{\Gamma}^{\mu}\left(x^{\alpha}, g_{\alpha \beta}\right)$, which are explicit functions of the coordinates and the metric, are introduced by replacing the harmonic conditions by $\Gamma^{\mu}=\hat{\Gamma}^{\mu}[17$. The reduced equations then become

$$
\hat{E}^{\mu \nu}=E^{\mu \nu}+\nabla^{(\mu} \hat{\Gamma}^{\nu)}-\frac{1}{2} g^{\mu \nu} \nabla_{\alpha} \hat{\Gamma}^{\alpha}=0 .
$$

The generalized harmonic conditions

$$
\mathcal{C}^{\mu}:=\Gamma^{\mu}-\hat{\Gamma}^{\mu}=0,
$$

are sufficient to ensure that Einstein's equations are satisfied. For this reason we refer to $\mathcal{C}^{\mu}$ as the constraints of the generalized harmonic formulation. They are related to the standard Hamiltonian and momentum constraints.

The Bianchi identities imply

$$
\nabla^{\alpha} \nabla_{\alpha} \mathcal{C}^{\mu}+R_{\alpha}^{\mu} \mathcal{C}^{\alpha}=0 .
$$

Because the hyperbolicity of (4.6) and (4.8) is unaffected by such gauge source functions, the Cauchy problem remains well-posed. The uniqueness of solutions to (4.8) thus ensures that the harmonic constraints $\mathcal{C}^{\mu}=0$ are satisfied in the domain of dependence of the initial Cauchy hypersurface $\mathcal{S}$ provided the initial data satisfy $\mathcal{C}^{\mu}=\partial_{t} \mathcal{C}^{\mu}=0$. It is straightforward to verify that Cauchy data on $\mathcal{S}$ which satisfy the Hamiltonian and momentum constraints $G_{\mu}^{t}=0$ and the initial condition $\mathcal{C}^{\mu}=0$ also satisfy $\partial_{t} \mathcal{C}^{\mu}=0$ on $\mathcal{S}$ by virtue of the generalized reduced harmonic equations (4.6).

The harmonic constraints (4.7) also imply equations (3.5) and (3.6) governing the time derivatives of the lapse and shift. As a result, in addition to the standard Cauchy data, i.e. the intrinsic metric and extrinsic curvature of $\mathcal{S}$ 
subject to the Hamiltonian and momentum constraints, the only other free initial data are the initial choices of lapse and shift.

Thus given Cauchy data $\gamma^{\mu \nu}$ and $\partial_{t} \gamma^{\mu \nu}$ that satisfies the Hamiltonian and momentum constraints in the gauge $\Gamma^{\mu}=0$, Cauchy data $\hat{\gamma}^{\mu \nu}$ and $\partial_{t} \hat{\gamma}^{\mu \nu}$ that satisfies the constraints in the gauge $\Gamma^{\mu}=\hat{\Gamma}^{\mu}$ can be obtained by setting $\hat{\gamma}^{\mu \nu}=\gamma^{\mu \nu}$ and $\partial_{t} \hat{g}^{i j}=\partial_{t} g^{i j}$ and then determining $\partial_{t} \hat{\gamma}^{t \alpha}$ from

$$
\frac{1}{\sqrt{-\hat{g}}} \partial_{\nu} \hat{\gamma}^{\mu \nu}=-\hat{\Gamma}^{\mu}
$$

Finally, $\partial_{t} \hat{\gamma}^{i j}$ is determined from

$$
\frac{1}{\sqrt{-\hat{g}}} \partial_{t} \hat{\gamma}^{i j}=\partial_{t} \hat{g}^{i j}+\frac{1}{2 \hat{g}} \hat{g}^{i j} \partial_{t} \hat{g}
$$

Here the calculation of $\partial_{t} \hat{g}$ can be expedited using the identity $g^{t t} g=h$, where $h=1 / \operatorname{det}\left(g^{i j}\right)$ is the determinant of the 3 -metric $h_{i j}$. Combined with the $t$-component of (4.9), this yields

$$
\frac{\partial_{t} \hat{g}}{2 \hat{g}}=\frac{\partial_{t} h}{h}+\frac{1}{g^{t t}}\left(\hat{\Gamma}^{t}+\frac{1}{\sqrt{-g}} \partial_{i} \gamma^{t i}\right)
$$

In order to examine whether a gauge source function can be effective in controlling the instability of the shifted gauge wave, we consider the simpler problem of spatially homogeneous Kerr-Schild metrics (3.9). First consider the choice

$$
\hat{\Gamma}^{\mu}=c t^{\mu}
$$

where $t^{\mu} \partial_{\mu}=\partial_{t}$ is the evolution vector. The resulting modification to (3.10) is

$$
\hat{E}^{\mu \nu}=\frac{1}{2}\left((1+F) F_{, t t}-F_{, t} F_{, t}+c F_{, t}\right) k^{\mu} k^{\nu}=0 .
$$

Because there is no modification to the nonlinear terms, there remain exponentially growing solutions of the form

$$
F=k e^{\lambda t}-1-\frac{c}{\lambda}
$$

Another example is the choice

$$
\hat{\Gamma}^{\mu}=\frac{c}{\sqrt{-g}}\left(\gamma^{t \mu}-\gamma_{[0]}^{t \mu}\right)
$$

where we use the notation $f_{[0]}=f\left(0, x^{i}\right)$. In the spatially homogeneous case, this choice leads via the constraints (4.7) to

$$
\partial_{t}\left(\gamma^{t \mu}-\gamma_{[0]}^{t \mu}\right)=-c\left(\gamma^{t \mu}-\gamma_{[0]}^{t \mu}\right)
$$

with solution $\gamma^{t \mu}=\gamma_{[0]}^{t \mu}$. Thus, if the constraints are satisfied, $\gamma^{t \mu}$ is forced to retain to its initial value, which has the potential advantage of warding off coordinate pathologies. If with the gauge forcing term (4.15) we make the homogeneous Kerr-Schild ansatz (3.9), then the reduced Einstein equations would require

$$
\hat{E}^{\mu \nu}=\frac{1}{2}\left((1+F) F_{, t t}-F_{, t} F_{, t}+c F_{[0]} F_{, t}\right) k^{\mu} k^{\nu}+\frac{c}{2} F_{, t}\left(\delta_{y}^{\mu} \delta_{y}^{\nu}+\delta_{z}^{\mu} \delta_{z}^{\nu}\right)=0 .
$$

Again the forcing term has only a linear effect but now it is inconsistent with the Kerr-Schild ansatz (except in the trivial case $F_{, t}=0$ ), so that the evolution would in general create other components in the metric and possibly excite other instabilities.

Other gauge source functions can be chosen which introduce nonlinear terms but we have not found an example which preserves the Kerr-Schild form (3.9). Consequently, an analytic analysis of their effect on the shifted gauge wave is difficult to carry out. It is clear from black hole simulations using harmonic coordinates that gauge forcing can play a helpful role [20, 21], but there are few general guidelines to go by. In the case of an analytic testbed, the addition of gauge forcing terms beyond those specified in the analytic solution can complicate the test if the resulting solution is not known. We have had no computational success in using gauge forcing terms to control instabilities in the shifted gauge wave test. 


\section{B. Constraint adjustment}

There is no extensive knowledge regarding the stability of solutions to the constraint systems (4.2) or (4.8). Of special importance to numerical evolution is whether constraint violating perturbations of the reduced Einstein equations can grow at a fast rate. This question is complicated by the fact that the reduced harmonic equations are not unique. They can be adjusted, without affecting their hyperbolicity, according to

$$
\begin{aligned}
\tilde{E}^{\mu \nu}: & =G^{\mu \nu}-\nabla^{(\mu} \mathcal{C}^{\nu)}+\frac{1}{2} g^{\mu \nu} \nabla_{\alpha} \mathcal{C}^{\alpha}+A^{\mu \nu} \\
& =E^{\mu \nu}+\nabla^{(\mu} \hat{\Gamma}^{\nu)}-\frac{1}{2} g^{\mu \nu} \nabla_{\alpha} \hat{\Gamma}^{\alpha}+A^{\mu \nu}=0
\end{aligned}
$$

provided the adjustment has the functional form

$$
A^{\mu \nu}=A^{\mu \nu}\left(x^{\alpha}, g^{\alpha \beta}, \partial_{\gamma} g^{\alpha \beta}, \mathcal{C}^{\alpha}\right)
$$

with $A^{\mu \nu}=0$ when $\mathcal{C}^{\alpha}=0$. The resulting constraint system (4.8) becomes

$$
\nabla^{\alpha} \nabla_{\alpha} \mathcal{C}^{\mu}+R_{\nu}^{\mu} \mathcal{C}^{\nu}-2 \nabla_{\nu} A^{\mu \nu}=0
$$

with the reduced Ricci tensor given by

$$
R^{\mu \nu}=\nabla^{(\mu} \mathcal{C}^{\nu)}-A^{\mu \nu}+\frac{1}{2} g^{\mu \nu} A
$$

The standard form of the reduced harmonic equations (3.7) differ from Fock's [1] harmonic formulation, on which the original version of the Abigel code was based [14], by the adjustment

$$
A^{\mu \nu}=\frac{1}{2 g} \Gamma^{(\mu} g^{\nu) \alpha} \partial_{\alpha} g-\frac{1}{4 g} g^{\mu \nu} \Gamma^{\alpha} \partial_{\alpha} g .
$$

In the absence of a general theory, computational experiments are necessary to determine the effect of a given adjustment. However, some clues can be provided by the following observations.

In the linear approximation, with unit lapse and zero shift, the adjustment [22]

$$
A^{\mu \nu}=-\lambda \mathcal{C}^{(\mu} \nabla^{\nu)} t, \quad \lambda>0
$$

leads to the constraint system

$$
\left(-\partial_{t}^{2}+\nabla^{2}\right) \mathcal{C}^{\mu}=\lambda\left(\partial_{t} \mathcal{C}^{\mu}+\delta_{t}^{\mu} \partial_{\nu} \mathcal{C}^{\nu}\right)
$$

The spatial components satisfy

$$
\left(-\partial_{\tau}^{2}+\nabla^{2}\right)\left(e^{\lambda t} \mathcal{C}^{i}\right)=0
$$

where $\tau=\left(e^{\lambda t}-1\right) / \lambda$. For physically reasonable boundary conditions, e.g. periodic boundary conditions, a solution $F$ of the wave equation (4.25) must have finite energy, so that

$$
\int|\nabla F|^{2} d x d y d z<K_{1}
$$

for some constant $K_{1}$. Consequently,

$$
\int\left|\nabla C^{i}\right|^{2} d x d y d z<K_{1} e^{-2 \lambda t}
$$

which implies that any initial error in the constraint $C^{i}$ must decay to a spatially constant solution $C_{\infty}^{i}(t)$. Such homogeneous solutions of (4.25) have the form $C_{\infty}^{i}=K_{2}+K_{3} e^{-\lambda t}$. By the analogous argument, any constraint violation in the time component $\mathcal{C}^{t}$ also decays to to a constant value. This result has previously been established for the case $\partial_{i} \mathcal{C}^{\mu} \neq 0$ using mode analysis [22].

Unfortunately, such constraint damping does not extend in a straightforward way to the nonlinear case, where in particular it can lead to the excitation of constraint preserving instabilities. For example, this adjustment does not 
preserve the Kerr-Schild metric type (3.9) so that in damping the instability (3.15) it can excite an instability of the type (3.2), which cannot be controlled by constraint adjustment. See Fig. [9 in Sec. DT for evidence of this behavior.

The exponential instability (3.12) satisfies the reduced harmonic equations but violates the harmonic constraints. Although we have found no way to control this instability with harmonic gauge source functions $\hat{\Gamma}^{\mu}$, it may be suppressed by the constraint adjustment

$$
A^{\mu \nu}=-\frac{c}{\sqrt{-g}} \mathcal{C}^{\alpha} \partial_{\alpha}\left(\sqrt{-g} g^{\mu \nu}\right), \quad c>0 .
$$

The reduced equations (4.18) (with $\hat{\Gamma}^{\mu}=0$ ) now give

$$
2 \sqrt{-g} \tilde{E}^{\mu \nu}=\left(F_{, t t}-F_{, t} F_{, t}+F F_{, t t}+2 c F_{t} F_{t}\right) k^{\mu} k^{\nu}=0
$$

with solutions

$$
F=\left(a_{1} t+a_{2}\right)^{1 /(2 c)}-1 .
$$

Thus the exponential growth has been removed. For the case $c=1 / 2$, in which $F$ grows only linearly in time, the effect of this adjustment is to remove the lowest differential order terms in $\mathcal{C}^{\mu}$ from [4.18), i.e.

$$
-\nabla^{(\mu} \mathcal{C}^{\nu)}+\frac{1}{2} g^{\mu \nu} \nabla_{\alpha} \mathcal{C}^{\alpha}+A^{\mu \nu}=\frac{1}{2}\left(-g^{\mu \alpha} \partial_{\alpha} \mathcal{C}^{\nu}-g^{\nu \alpha} \partial_{\alpha} \mathcal{C}^{\mu}\right)
$$

The growth rate can be completely eliminated by the adjustment

$$
A^{\mu \nu}=-\frac{b}{2} g^{t t} k^{(\mu} \mathcal{C}^{\nu)}, \quad b>0 .
$$

Then (4.18) gives

$$
2 \sqrt{-g} \tilde{E}^{\mu \nu}=\left((1+F)\left(F_{, t t}+b F_{, t}\right)-F_{, t} F_{, t}\right) k^{\mu} k^{\nu}=0
$$

with the strongly damped solution

$$
1+F=a_{1} \exp \left(a_{2} e^{-b t}\right) .
$$

The same strongly damped behavior (4.34) follows from the adjustment

$$
A^{\mu \nu}=\frac{b \mathcal{C}^{\alpha} \nabla_{\alpha} t}{e_{\rho \sigma} \mathcal{C}^{\rho} \mathcal{C}^{\sigma}} \mathcal{C}^{\mu} \mathcal{C}^{\nu}, \quad b>0
$$

where

$$
e_{\rho \sigma}=g_{\rho \sigma}-\frac{2}{g^{t t}}\left(\nabla_{\rho} t\right) \nabla_{\sigma} t
$$

is the natural metric of signature $(++++)$ associated with the Cauchy slicing. Here 4.35 has the advantage over (4.32) that it has a geometric construction which is independent of the Kerr-Schild form of the metric.

The adjustments (4.28) and especially (4.35) lead to improved performance in the shifted gauge wave tests described in Sec VI Other adjustments can be designed to introduce lowest differential order terms which act as a repulsive potential in the wave equation (4.20). For example, consider the adjustment

$$
A^{\mu \nu}=-k g^{\mu \nu} \Phi^{2}, k>0 .
$$

where $\Phi=\mathcal{C}^{\alpha} \mathcal{C}_{\alpha}$ Then, after contraction with $\mathcal{C}_{\mu}$, 4.20) gives

$$
\nabla_{\alpha} \nabla^{\alpha} \Phi-2 k \Phi^{3}=\mathcal{F}
$$

where $\mathcal{F}$ vanishes when $\nabla_{\alpha} \mathcal{C}^{\beta}=0$. By choosing $k$ to be large, this repulsive $\Phi^{4}$ potential might at the least constrain $\mathcal{C}^{\alpha}$ to be a null vector. 


\section{FINITE DIFFERENCE IMPLEMENTATION}

A first differential order formalism is useful for applying the well developed theory of symmetric hyperbolic systems but in a numerical code it introduces extra variables and their associated nonphysical constraints. For this reason, we base our code on the natural second order form of the quasilinear wave equations which comprise the reduced harmonic system (4.18). They are finite differenced in the flux conservative form

$$
2 \sqrt{-g} \tilde{E}=\partial_{\alpha}\left(g^{\alpha \beta} \partial_{\beta} \gamma^{\mu \nu}\right)-S^{\mu \nu}=0
$$

where $S^{\mu \nu}$ is comprised of (nonlinear) first derivative terms that do not enter the principle part.

Numerical evolution is implemented on a spatial grid $\left(x_{I}, y_{J}, z_{K}\right)=(I h, J h, K h), 0 \leq(I, J, K) \leq N$, with uniform spacing $h$, on which a field $f\left(t, x^{i}\right)$ is represented by its grid values $f_{[I, J, K]}(t)=f\left(t, x_{I}, y_{J}, z_{K}\right)$. The time integration is carried out by the method of lines using a 4 th order Runge-Kutta method. We introduce the standard finite difference operators $D_{0 i}$ and $D_{ \pm i}$ according to the examples

$$
\begin{aligned}
D_{0 x} f_{I, J} & =\frac{1}{2 h}\left(f_{I+1, J}-f_{I-1, J}\right) \\
D_{+x} f_{I, J} & =\frac{1}{h}\left(f_{I+1, J}-f_{I, J}\right) \\
D_{-y} f_{I, J} & =\frac{1}{h}\left(f_{I, J}-f_{I, J-1}\right) ;
\end{aligned}
$$

the translation operators $T_{ \pm i}$ according to the example

$$
T_{ \pm x} f_{I, J}=f_{I \pm 1, J}
$$

and the averaging operators $A_{ \pm i}$ and $A_{0 i}$, according to the examples

$$
\begin{gathered}
A_{ \pm x} f_{I, J}=\frac{1}{2}\left(T_{ \pm x}+1\right) f_{I, J} \\
A_{0 x} f_{I, J}=\frac{1}{2}\left(T_{+x}+T_{-x}\right) f_{I, J} .
\end{gathered}
$$

Standard centered differences $D_{0 i}$ are used to approximate the first derivative terms in (5.1) comprising $S^{\mu \nu}$.

We will describe the finite difference techniques for the principle part of (5.1) in terms of the scalar wave equation

$$
\partial_{\alpha}\left(g^{\alpha \beta} \partial_{\beta} \Phi\right)=0 .
$$

Note that the principle part of the linearization of (5.1) gives rise to (5.3) for each component of $\gamma^{\mu \nu}$. The nonvanishing shift leads to mixed space-time derivatives $\partial_{t} \partial_{i}$ which complicates the use of standard explicit algorithms for the wave equation. This problem has been addressed in [15, 23, 24, 25]. Here we base our work on an evolution algorithm shown to be stable for a model 1-dimensional wave equation with shift [15]. Provided $g^{i j}$ is positive definite, as is the case when the shift is subluminal (i.e. when the evolution vector $t^{\alpha} \partial_{\alpha}=\partial_{t}$ is timelike), this algorithm has the summation by parts (SBP) property that gives rise to an energy estimate for (5.3). SBP algorithms have proved effective in other numerical relativity codes [26, 27, 28].

The algorithm we use is designed to obey semi-discrete versions of two conservation laws obeyed by (5.3). These govern the monopole quantity

$$
Q=-\int_{V} g^{t \alpha} \Phi_{, \alpha} d V
$$

and the energy

$$
E=\frac{1}{2} \int_{V}\left(-g^{t t} \Phi_{, t}^{2}+g^{i j} \Phi_{, i} \Phi_{, j}\right) d V,
$$

where $d V=d x d y d z$. By assumption, the $t=$ const Cauchy hypersurfaces are spacelike so that $-g^{t t}>0$. We also assume in the following that $g^{i j}$ is positive definite (subluminal shift) so that $E$ provides a norm. Note that in the gravitational case, where $\Phi$ represents $\gamma^{\mu \nu}$, there are 10 quantities $Q^{\alpha \beta}$ corresponding to (5.4), which have monopole, dipole or quadrupole transformation properties depending on the choice of indices. 
For periodic boundary conditions (or in the absence of a boundary), (5.3) implies strict monopole conservation $Q_{, t}=0$; and, when the coefficients of the wave operator are frozen in time, i.e. when $\partial_{t} g^{\alpha \beta}=0$, (5.3) implies strict energy conservation $E_{, t}=0$. In the time-dependent, boundary-free case,

$$
E_{, t}=\frac{1}{2} \int_{V}\left(g_{, t}^{\alpha \beta} \Phi_{, \alpha} \Phi_{, \beta}\right) d V .
$$

which readily provides an estimate of the form

$$
E_{, t}<k E
$$

for some $k$ independent of the initial data for $\Phi$. Thus the norm is bounded relative to its initial value at $t=0$ by

$$
E<A E_{0} e^{k t}
$$

The most restrictive value of $k$ depends upon the ratios of the norms of the quadratic forms defined by the integrands in (5.5) and (5.6).

For the present purpose, it suffices to describe the finite difference evolution algorithm in the 2-dimensional case with periodic boundary conditions $f_{0, J}=f_{N, J}, f_{I, 0}=f_{I, N}$. We define the semi-discrete versions of (5.4) and (5.5) as

$$
Q=h^{2} \sum_{(I, J)=1}^{N}\left(-g^{t t} \Phi_{, t}-g^{t i} D_{0 i} \Phi\right) .
$$

and

$$
E=h^{2} \sum_{(I, J)=1}^{N} \mathcal{E}
$$

where

$$
\begin{aligned}
\mathcal{E}= & -\frac{1}{2} g^{t t} \Phi_{, t}^{2}+\frac{1}{4}\left(A_{+x} g^{x x}\right)\left(D_{+x} \Phi\right)^{2}+\frac{1}{4}\left(A_{-x} g^{x x}\right)\left(D_{-x} \Phi\right)^{2} \\
& +\frac{1}{4}\left(A_{+y} g^{y y}\right)\left(D_{+y} \Phi\right)^{2}+\frac{1}{4}\left(A_{-y} g^{y y}\right)\left(D_{-y} \Phi\right)^{2}+g^{x y}\left(D_{0 x} \Phi\right) D_{0 y} \Phi
\end{aligned}
$$

The energy provides a norm on the discretized system, i.e. $E=0$ implies $\Phi_{, t}=D_{ \pm i} \Phi=0$ (provided the grid spacing $h$ is sufficiently small to justify regrouping of terms into perfect squares as in the continuum case).

The simplest second order approximation to (5.3) which reduces in the 1-dimensional case to the SBP algorithm presented in [15] is

$$
W:=-\partial_{t}\left(g^{t t} \partial_{t} \Phi\right)-\partial_{t}\left(g^{t i} D_{0 i} \Phi\right)-D_{0 i}\left(g^{i t} \partial_{t} \Phi\right)-\mathcal{D}_{g}^{2} \Phi=0
$$

where

$$
\begin{aligned}
\mathcal{D}_{g}^{2} \Phi & =\frac{1}{2} D_{+x}\left(\left(A_{-x} g^{x x}\right) D_{-x} \Phi\right)+\frac{1}{2} D_{-x}\left(\left(A_{+x} g^{x x}\right) D_{+x} \Phi\right) \\
& +\frac{1}{2} D_{+y}\left(\left(A_{-y} g^{y y}\right) D_{-y} \Phi\right)+\frac{1}{2} D_{-y}\left(\left(A_{+y} g^{y y}\right) D_{+y} \Phi\right) \\
& +D_{0 x}\left(g^{x y} D_{0 y} \Phi\right)+D_{0 y}\left(g^{x y} D_{0 x} \Phi\right)
\end{aligned}
$$

It follows immediately from the flux conservative form of $W$ that $Q_{, t}=0$ for the case of periodic boundary conditions. In order to establish the SBP property we consider the frozen coefficient case $\partial_{t} g^{\alpha \beta}=0$. Then a straightforward calculation gives

$$
\begin{aligned}
\mathcal{E}_{t}-\Phi_{t} W & =\frac{1}{2} D_{+i}\left(g^{t i} \Phi_{t} T_{-i} \Phi_{t}+\Phi_{t} T_{-i}\left(g^{t i} \Phi_{t}\right)\right) \\
& +\frac{1}{2} D_{+x}\left(\left(A_{-x} g^{x x}\right)\left(D_{-x} \Phi\right) T_{-x} \Phi_{t}\right)+\frac{1}{2} D_{-x}\left(\left(A_{+x} g^{x x}\right)\left(D_{+x} \Phi\right) T_{+x} \Phi_{t}\right)
\end{aligned}
$$




$$
\begin{aligned}
& +\frac{1}{2} D_{+y}\left(\left(A_{-y} g^{y y}\right)\left(D_{-y} \Phi\right) T_{-y} \Phi_{t}\right)+\frac{1}{2} D_{-y}\left(\left(A_{+y} g^{y y}\right)\left(D_{+y} \Phi\right) T_{+y} \Phi_{t}\right) \\
& +\frac{1}{2} D_{+x}\left(\Phi_{t} T_{-x}\left(g^{x y} D_{0 y} \Phi\right)+g^{x y}\left(D_{0 y} \Phi\right) T_{-x} \Phi_{t}\right) \\
& +\frac{1}{2} D_{+y}\left(\Phi_{t} T_{-y}\left(g^{x y} D_{0 x} \Phi\right)+g^{x y}\left(D_{0 x} \Phi\right) T_{-y} \Phi_{t}\right) .
\end{aligned}
$$

Because each term in (5.14) is a total $D_{ \pm i}$, it follows (for periodic boundary conditions) that $E_{, t}=0$ when $W=0$ is satisfied. When the coefficients of the wave operator are time dependent, an energy estimate can be established analogous to (5.8) for the continuum case.

We also consider a modification of the algorithm (5.8) by introducing extra averaging operators according to

$$
\hat{W}:=-\partial_{t}\left(g^{t t} \partial_{t} \Phi\right)-\partial_{t}\left(g^{t i} D_{0 i} \Phi\right)-D_{-i}\left(\left(A_{+i} g^{i t}\right)\left(A_{+i} \partial_{t} \Phi\right)\right)-{\hat{D_{g}}}^{2} \Phi=0,
$$

with

$$
\begin{aligned}
\hat{D}_{g}^{2} \Phi & =\frac{1}{2} D_{+x}\left(\left(A_{-x} g^{x x}\right) D_{-x} \Phi\right)+\frac{1}{2} D_{-x}\left(\left(A_{+x} g^{x x}\right) D_{+x} \Phi\right) \\
& +\frac{1}{2} D_{+y}\left(\left(A_{-y} g^{y y}\right) D_{-y} \Phi\right)+\frac{1}{2} D_{-y}\left(\left(A_{+y} g^{y y}\right) D_{+y} \Phi\right) \\
& +D_{-x}\left(\left(A_{+x} g^{x y}\right)\left(A_{+x} D_{0 y} \Phi\right)\right)+D_{-y}\left(\left(A_{+y} g^{x y}\right)\left(A_{+y} D_{0 x} \Phi\right)\right) .
\end{aligned}
$$

It is easy to verify that $\hat{W}=W+O\left(h^{2}\right)$ and both $W$ and $\hat{W}$ are constructed from the same stencil of gridpoints. Although $\hat{W}$ does not obey the exact SBP property with respect to the energy (5.11), the experiments in Sec. VI] show that it leads to significantly better performance for the shifted gauge wave test.

For the time discretization, we apply the method of lines to the large system of ordinary differential equations

$$
\boldsymbol{\Phi}_{, t t}=\frac{1}{h} \mathbf{A} \boldsymbol{\Phi}_{, t}+\frac{1}{h^{2}} \mathbf{B} \boldsymbol{\Phi} .
$$

obtained from the spatial discretization. Introducing

$$
\mathbf{\Phi}_{, t}=\frac{1}{h} \mathbf{T},
$$

we obtain the first order system

$$
\left(\begin{array}{l}
\mathbf{T} \\
\mathbf{\Phi}
\end{array}\right)_{t}=\frac{1}{h}\left(\begin{array}{cc}
\mathbf{B} & \mathbf{A} \\
\mathbf{I} & \mathbf{0}
\end{array}\right)\left(\begin{array}{c}
\mathbf{T} \\
\mathbf{V}
\end{array}\right)
$$

We solve the system numerically using a 4th order Runge-Kutta time integrator.

Dissipation can be added by modifying (5.19) according to

$$
\begin{aligned}
& \mathbf{T}_{t} \rightarrow \mathbf{T}_{t}+\epsilon_{T} h^{3} \mathcal{D}_{g}^{2} \mathcal{D}_{g}^{2} \mathbf{T}, \\
& \mathbf{\Phi}_{t} \rightarrow \mathbf{\Phi}_{t}+\epsilon_{\Phi} h^{3} \mathcal{D}_{g}^{2} \mathcal{D}_{g}^{2} \mathbf{\Phi} .
\end{aligned}
$$

The $W$ algorithm is unstable when $g^{i j}$ is not positive-definite or, equivalently, when the evolution direction $t^{\alpha}$ is superluminal (spacelike). There are alternative evolution algorithms for the second differential order 1D wave equation which are stable in the superluminal case [15, 24]. These algorithms have important application to the black hole excision problem in treating the region inside the event horizon. However they have no advantage in the shifted gauge wave test because the evolution is superluminal only for amplitudes $A>1$ for which the spacelike nature of the Cauchy hypersurfaces breaks down. These alternative algorithms remain stable in the subluminal case but they are not as accurate as the $W$ algorithm because they involve a wider stencil of grid points. Nevertheless, it is useful to compare their accuracy with the $W$ algorithm. (See Fig. [7 in Sec. VI)

For that purpose we introduce the simplest generalization of the $V$ algorithm considered in [15] to the 3D case. It is related to the $W$ algorithm (5.12) by

$$
\left.V=W+\left(\mathcal{D}_{g}^{2}-\mathcal{D}_{h}^{2}\right)\right) \Phi-\mathcal{D}_{\beta, 2}^{2} \Phi
$$


where $\mathcal{D}_{h}^{2}$ is defined as in (5.13) with $g^{i j}$ replaced by the spatial 3-metric

$$
h^{i j}=g^{i j}-\frac{g^{t i} g^{t j}}{g^{t t}} .
$$

and the shift terms are finite differenced by

$$
\mathcal{D}_{\beta, 2}^{2} \Phi=D_{0 x}\left(\frac{g^{t x} g^{t x}}{g^{t t}} D_{0 x} \Phi\right)+D_{0 y}\left(\frac{g^{t y} g^{t y}}{g^{t t}} D_{0 y} \Phi\right)+D_{0 x, 2 h}\left(\frac{g^{t x} g^{t y}}{g^{t t}} D_{0 y, 2 h} \Phi\right)+D_{0 y, 2 h}\left(\frac{g^{t x} g^{t y}}{g^{t t}} D_{0 x, 2 h} \Phi\right),
$$

with, for example,

$$
D_{0 x, 2 h} f(x, y)=\frac{f(x+2 h, y)-f(x-2 h, y)}{4 h}=\left(1+\frac{h^{2}}{2} D_{+x} D_{-x}\right) D_{0 x} f .
$$

Equivalently, $V$ and $W$ are related by the $O\left(h^{2}\right)$ terms

$$
\begin{aligned}
V & =W-\frac{h^{2}}{4}\left(D_{+x} D_{-x} \frac{g^{t x} g^{t x}}{g^{t t}} D_{+x} D_{-x}+D_{+y} D_{-y} \frac{g^{t y} g^{t y}}{g^{t t}} D_{+y} D_{-y}\right) \Phi \\
& -\frac{h^{2}}{2}\left(D_{0 x, 2 h} \frac{g^{t x} g^{t y}}{g^{t t}} D_{+y} D_{-y} D_{0 y}+D_{0 y, 2 h} \frac{g^{t x} g^{t y}}{g^{t t}} D_{+x} D_{-x} D_{0 x}\right. \\
& \left.+D_{+x} D_{-x} D_{0 x} \frac{g^{t x} g^{t y}}{g^{t t}} D_{0 y}+D_{+y} D_{-y} D_{0 y} \frac{g^{t x} g^{t y}}{g^{t t}} D_{0 x}\right) \Phi .
\end{aligned}
$$

We also consider the modification

$$
\hat{V}=\hat{W}+\left(\mathcal{D}_{g}^{2}-\mathcal{D}_{h}^{2}\right) \Phi-\mathcal{D}_{\beta, 2}^{2} \Phi .
$$

\section{TESTS OF THE EVOLUTION ALGORITHM}

We test the evolution code using the gauge wave and shifted gauge wave testbeds with periodic boundary conditions and amplitude $A=.5$. Test results for the standard AwA gauge wave with amplitudes $A=.01$ and $A=.1$ using an earlier version of the Abigel code can be found at the Pitt numerical relativity web site [29]. (Those tests were performed using an iterated Crank-Nicholson time integrator on a code similar to the $\hat{W}$ algorithm (5.15) with the constraint adjustments (4.22) and (4.28) with $c=.5$ ). The present code shows similar performance for the standard AwA gauge wave test.

The tests are run on grids with $N=50 \rho$ zones, where $\rho=(1,2,4)$. We use the $\ell_{\infty}$ norm to measure the error

$$
\mathcal{E}=\left\|\Phi_{\rho}-\Phi_{\text {ana }}\right\|_{\infty}
$$

in a grid function $\Phi_{\rho}$ with known analytic value $\Phi_{a n a}$. We measure the convergence rate at time $t$

$$
r(t)=\log _{2}\left(\frac{\left\|\Phi_{2}-\Phi_{\text {ana }}\right\|}{\left\|\Phi_{4}-\Phi_{\text {ana }}\right\|}\right)
$$

using the $\rho=2$ and $\rho=4$ grids $(N=100$ and $N=200)$. It is also convenient to graph the rescaled error

$$
\mathcal{E}_{\rho}=\frac{\rho^{2}}{16}\left\|\Phi_{\rho}-\Phi_{\text {ana }}\right\|_{\infty}
$$

which is normalized to the $\rho=4$ grid.

\section{A. Tests for the gauge wave without shift}

Figures 1 and 2 plot our results for the rescaled error $\mathcal{E}_{\rho}$ in $g_{x x}$, for $\rho=1,2,4(N=50,100,200)$, for the 1D and 2D gauge wave tests with the gauge wave with amplitude $A=.5$ using the bare $\hat{W}$ algorithm (5.15) (no additional gauge forcing, constraint adjustment or dissipation). The $1 \mathrm{D}$ runs were stopped at $t=1000$. At that time the absolute 
error for the coarsest grid was unacceptably large (over 100\%) but the error for the $\rho=4$ grid was only $\sim 20 \%$. We found the convergence rates

$$
r(50)=2.019, \quad r(500)=1.677
$$

for the error in $g_{x x}$, measured at $\mathrm{t}=50$ and $\mathrm{t}=500$ (corresponding to 50 and 500 grid crossing times). For computational economy, the $2 \mathrm{D}$ runs, where the wave propagates along a diagonal in the $(x, y)$ plane, were stopped at $t=100$. We measured the convergence rates

$$
r(10)=2.084, \quad r(100)=1.568
$$

at $t=10$ and $t=100$. Tests runs with the $W$ algorithm (5.12) showed similar performance although for the coarsest grid the $\hat{W}$ algorithm had noticeably smaller error, presumably as a result of the extra averaging operators.

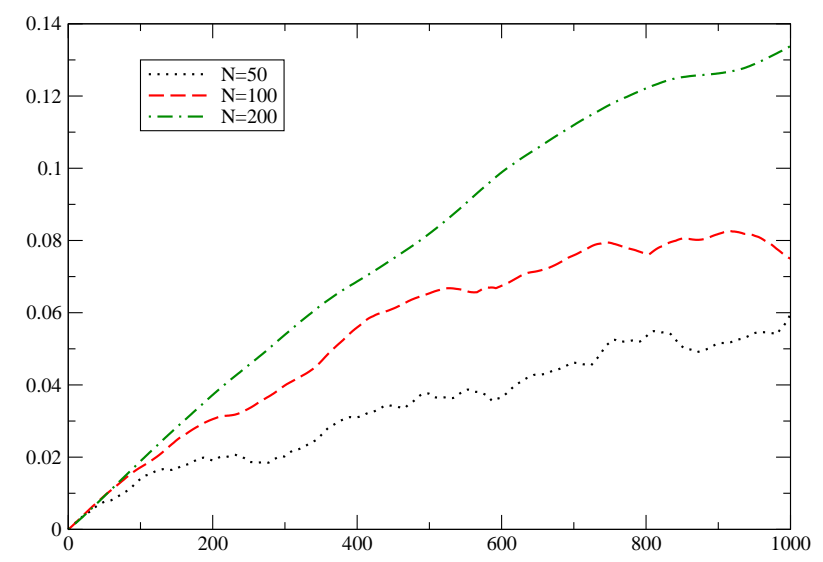

FIG. 1: Plot of the rescaled error $\mathcal{E}_{\rho}(t)$ for the $1 \mathrm{D}$ gauge wave.

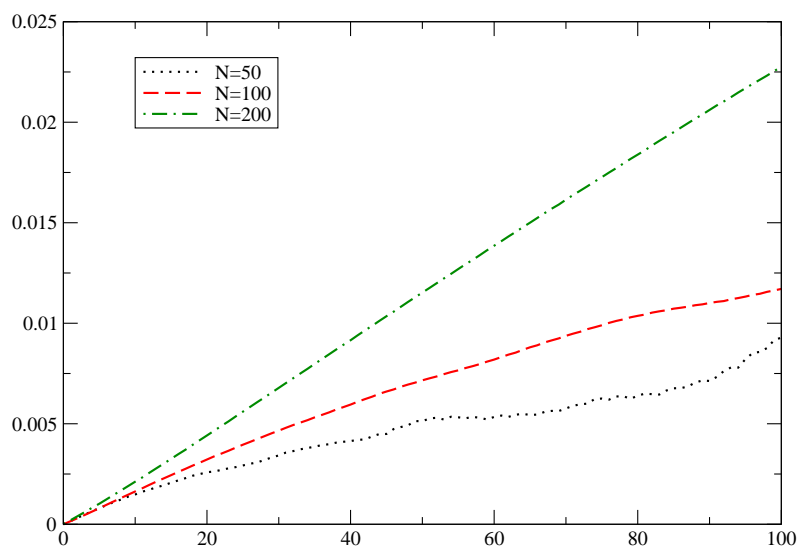

FIG. 2: Plot of the rescaled error $\mathcal{E}_{\rho}(t)$ for the $2 \mathrm{D}$ gauge wave.

The bare $W$ or $\hat{W}$ algorithms give excellent results for these gauge wave tests. No appreciable improvement is attained by using the gauge source functions or constraint adjustments discussed in Sec. IV or by using dissipation. It should be emphasized that this success is due to the discrete conservation laws built into the algorithm. In comparison, Fig. 3 shows snapshots of $g_{t t}(x)$ for a simulation of the gauge wave with $A=.5$ using an earlier version of the code without these conservative properties. The snapshots are almost perfect matches to the exponentially unstable mode (3.2). Table@ summarizes our results. 


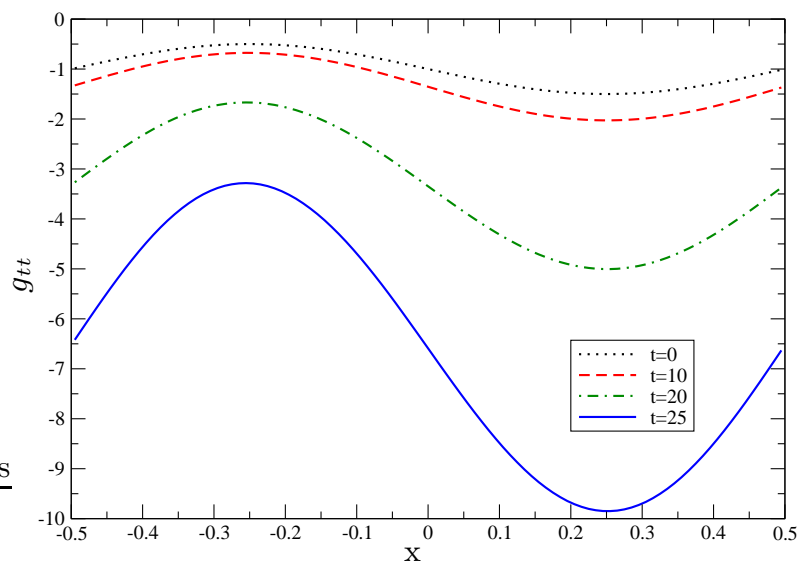

FIG. 3: Snapshots of $g_{t t}(x)$ for the 1D gauge wave without shift obtained with an earlier version of the code without conservative properties, on an $N=100$ grid. The error almost exactly matches the unstable mode [3.2].

\begin{tabular}{|c|c|}
\hline Test & Performance \\
\hline \hline Non-conservative algorithm, 1D & Exponentially unstable mode (Fig. 3 ) \\
\hline$W$ versus $\hat{W}$ algorithm, 1D & $\hat{W}$ has smaller error, due to extra averaging operators \\
\hline$W$ versus $\hat{W}$ algorithm, 2D & $\hat{W}$ has smaller error \\
\hline$\hat{W}$ algorithm, convergence, 1D & Long term second order convergence (Fig. 10 \\
\hline$\hat{W}$ algorithm, convergence, 2D & Long term second order convergence (Fig. 2 \\
\hline
\end{tabular}

TABLE I: Summary of tests performed for the gauge wave without shift, with amplitude $A=0.5$. Gauge forcing terms, constraint adjustments and dissipation all gave no appreciable improvement.

\section{B. Tests for the Shifted Gauge Wave}

For the shifted gauge wave tests we first consider the bare algorithms (no additional constraint gauge forcing, adjustment or dissipation). In this case, the $\hat{W}$ algorithm shows marked improvement over the $W$ algorithm. This

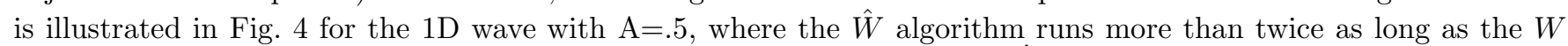
algorithm with equal error. For that reason we confine our attention to the $\hat{W}$ algorithm in the remaining tests. With the $\hat{W}$ algorithm, at $t=50$ we measured a convergence rate of

$$
r(50)=2.135
$$

in the error of $g_{x x}$ for the shifted gauge wave. The error is plotted in Fig. 囵 for grids with $N=50,100$ and 200.

The major error in the simulations with the bare algorithms is in the long wavelength exponential mode (3.12), which cause the runs with the coarser grids to crash at an early time. This is evident from the series of snapshots of $g_{t t}(x)$ shown in Fig. 6 for the $N=100$ grid. At $t \approx 200$, the long wavelength error triggers the superluminal instability of the $W$ algorithm as $g_{t t} \rightarrow 0$ at its peak value. The snapshots of $g_{t t}(x)$ closely match the unstable constraint violating mode (3.12). The exponential term in (3.12) introduces a positive error in $g_{t t}$ but a negative error in $g^{t t}$ so that the Cauchy hypersurfaces remain spacelike as $g_{t t} \rightarrow 0$. As a result, this error does not trigger an instability in the $V$ algorithm which is stable in the superluminal case, as discussed in Sec. $\nabla$

Tests of the bare $V$ and $\hat{V}$ algorithms (5.21) and (5.26) with the 1D shifted gauge wave gave convergent results but the error was considerably larger than with the bare $W$ and $\hat{W}$ algorithms. Fig. 7 compares the $\ell_{\infty}$ error in $g_{x x}$ between the $\hat{V}$ and the $\hat{W}$ algorithms. The $\hat{V}$ algorithm remains stable well beyond the time $t \approx 200$ when the error brings it into the superluminal regime. However, at $t \approx 200$ the error in the simulation is $\approx 100 \%$ and has begun to grow rapidly, while the error in the $\hat{W}$ algorithm is still small. Thus the trade-off for the stability of the $V$ algorithm in the superluminal regime is its relative inaccuracy in the subluminal regime compared to the $W$ algorithm. These results support the strategy adopted in [15] for a model black hole excision problem, in which the $V$-algorithm is used 


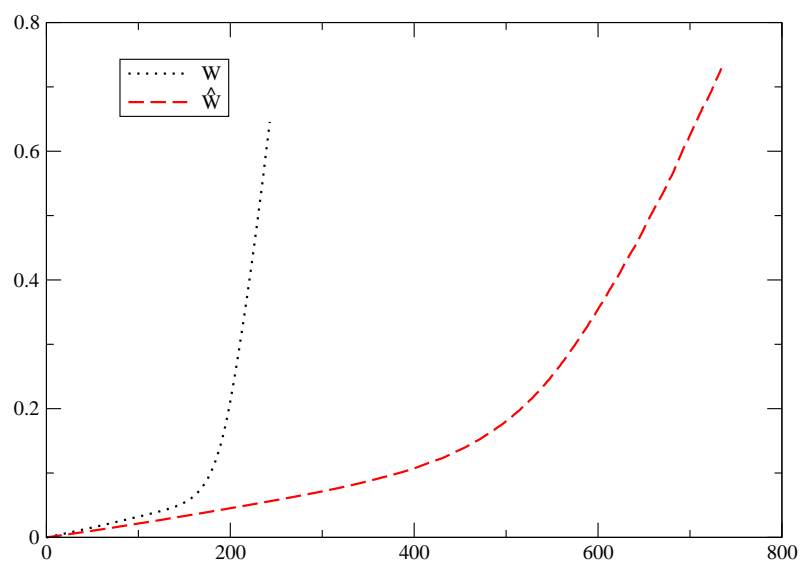

FIG. 4: Comparison plots of the unscaled error $\mathcal{E}(t)$ in $g_{x x}$ obtained with the bare $W$ and $\hat{W}$ algorithms for the $1 \mathrm{D}$ shifted gauge wave test, on an $\mathrm{N}=200$ grid. Although the $W$ algorithm has the SBP property, the additional averaging operators in the $\hat{W}$ algorithm lead to increased accuracy in this nonlinear test.

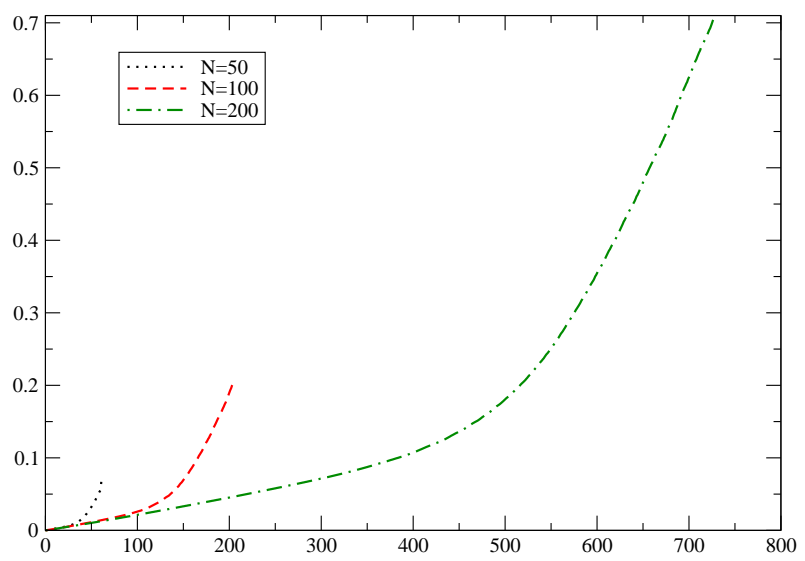

FIG. 5: Plot of the rescaled error $\mathcal{E}_{\rho}(t)$ in $g_{x x}$ obtained with the bare $\hat{W}$ algorithm for the $1 \mathrm{D}$ shifted gauge wave test.

in the inner region containing the horizon and the $W$-algorithm is used in an outer region extending to the outer boundary, with the two algorithms blended in a transition region.

We next consider tests with constraint adjustment. Excellent performance of the $\hat{W}$ algorithm results from the constraint adjustment (4.28). The error in $g_{x x}$ for the $N=100$ grid is plotted in Fig. 8 for this adjustment with $c=0, c=.5$ and $c=1$. As evident from the plots, the $c=1$ run remains under control at $t=1000$ whereas the unadjusted run crashes at $t \approx 200$. This adjustment with $c=0.5$ also leads to improvement over the unadjusted case, but the results are much more modest.

The constraint damping adjustment (4.23) leads to some improvement over the unadjusted case but the results are not nearly as effective as the adjustment (4.28). The analysis leading to the decaying behavior 4.25) in the linear regime does not lead to substantially improved performance in this nonlinear test. As shown in Fig. 9, the error obtained with the constraint damped $\hat{W}$ algorithm, with $\lambda=1$, is slightly smaller at early times than for the undamped case. However, the error for the damped case then goes through large oscillations and is roughly the same as for the undamped case at $t=200$. Although the constraint damped case runs longer, the highly oscillatory behavior produces unacceptably large error. The constraints $\mathcal{C}^{\mu}$ exhibit similar oscillation, indicating a coupling to a constraint preserving mode. Our experiments indicate significant improvement cannot be obtained by choosing other values of the damping coefficient $\lambda$ or by replacing $t^{\alpha}$ in (4.23) by another timelike vector, such as the vector $\nabla^{\alpha} t$ normal to the Cauchy hypersurfaces. (We obtained slightly better results for $t^{\alpha}$.) The addition of numerical dissipation (5.20) prolongs the run but does not control the large oscillations produced by the constraint damping and does not lead to any substantial increase in accuracy. Fig. 9 shows the effect of dissipation with $\epsilon_{\Phi}=.1$.

In addition to the $c$-adjustment (4.28), we found that the $b$-adjustment (4.35) also gives excellent performance in the shifted gauge wave test. Fig. 10 compares the error, on an $N=200$ grid, obtained using the $\hat{W}$ algorithm when adjusted by (4.35) with $b=1$ and when adjusted by (4.28) with $c=1$. Both adjustments are effective in controlling 


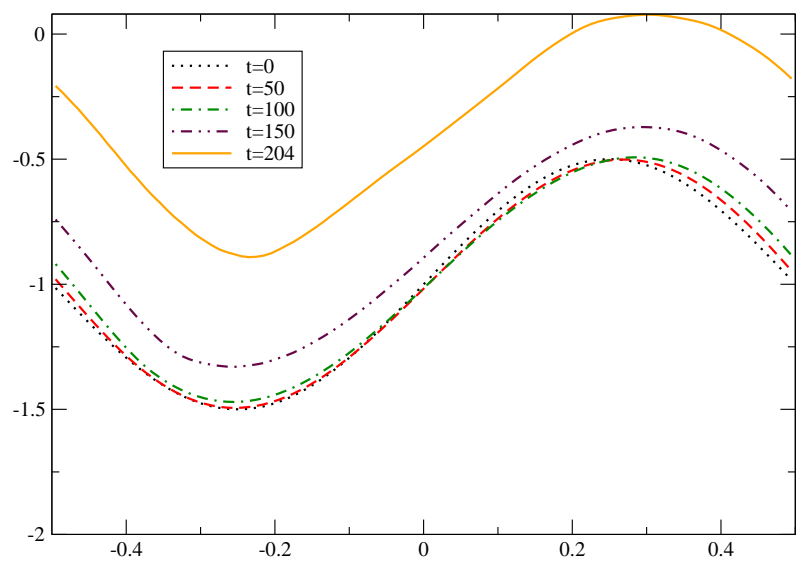

FIG. 6: Snapshots of $g_{t t}(x)$ of the shifted gauge wave obtained with the bare $\hat{W}$ algorithm on an $N=100$ grid. The behavior closely matches the unstable mode (3.12). At $t \approx 200$, the superluminal instability of the $W$ algorithm is triggered.

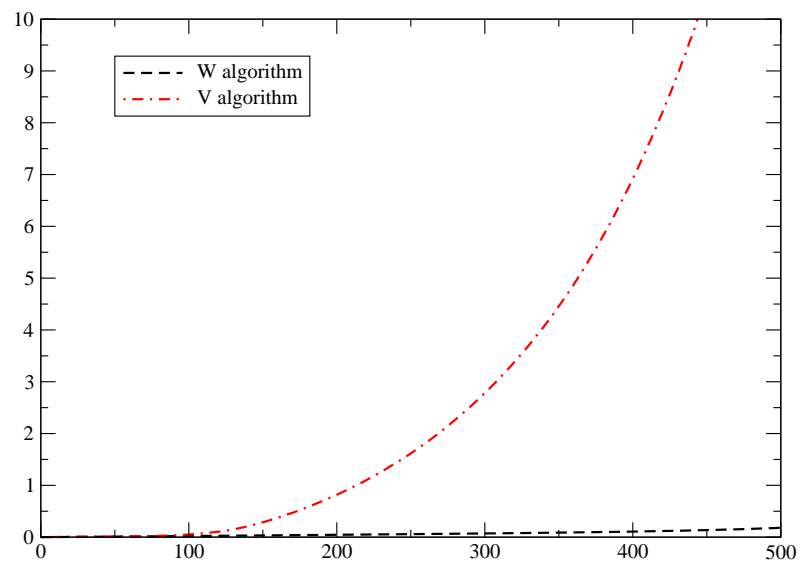

FIG. 7: Plots of the unscaled error $\mathcal{E}(t)$ in $g_{x x}$ on an $N=200$ grid comparing the bare $\hat{W}$ and $\hat{V}$ algorithms for the $1 \mathrm{D}$ shifted gauge wave.

the unstable mode, which is evident in the graph for $b=c=0$. The error for the two constraint adjusted cases looks very similar, although a closer inspection shows small oscillations in the $c=1$ graph, of the sort evident in Fig. 8 on an $N=100$ grid, but which do not appear for $b=1$.

Our experiments with gauge forcing terms, such as 4.15], gave no significant improvement in performance. However, this is likely a feature of the shifted gauge wave test for which harmonic coordinates do not encounter any pathologies. As reported in 20, 21], we expect gauge forcing terms to be essential for the long term simulation of black holes.

We found the 1D shifted gauge wave tests to be very effective in determining those algorithms which would give good performance in the 2D test. For that reason, we limit our presentation of $2 \mathrm{D}$ test results for the $\hat{W}$ algorithm with the $b=1$ constraint adjustment (4.35). Fig. 11] shows the rescaled error obtained using $N=50, N=100$ and $N=200$ grids. The convergence rate $r(100)=2.050$ was measured at $t=100$. Table $\Pi$ summarizes our results.

\section{CONCLUSION}

Computational fluid dynamics developed into a mature field only after a long struggle during which progress of lasting value emerged from the discriminating use of model problems and standardized tests. An important potential payoff of numerical relativity is the simulation of gravitational wave sources but sound methodology and testing are essential in developing trustworthy codes. The gauge wave tests presented here provide strong caution that a stable convergent code is not sufficient to obtain long term simulations, as exhibited by the problems introduced by long wavelength unstable modes in Figs. Band 6 In the absence of analytic solutions this caution extends to the simulation of binary black holes. It would be of value to compare the results reported here for the shifted gauge wave test with the performance of codes based upon different hyperbolic reductions of the Einstein equations and different finite 


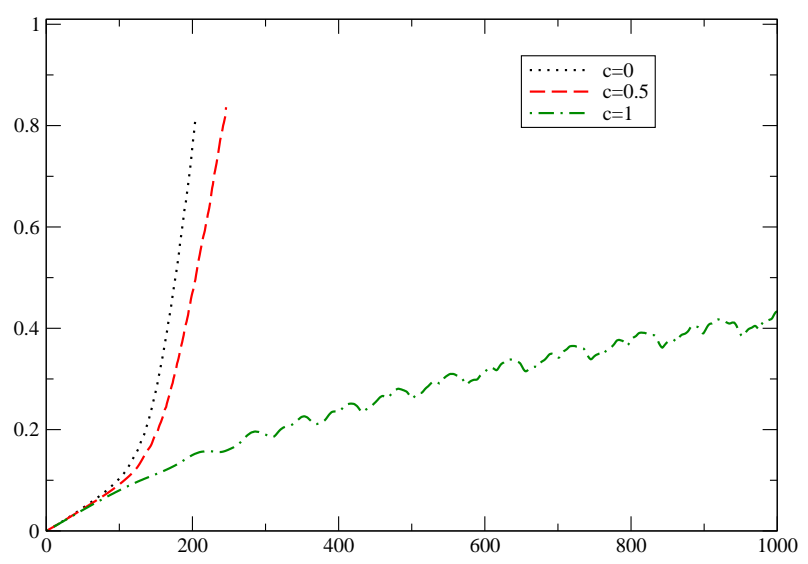

FIG. 8: The unscaled error $\mathcal{E}(t)$ in the component $g_{x x}$, on an $N=100$ grid, using the constraint adjustment 4.28 in the $1 \mathrm{D}$ shifted gauge wave test. The adjustment with $c=1$ very effectively suppresses the instability.

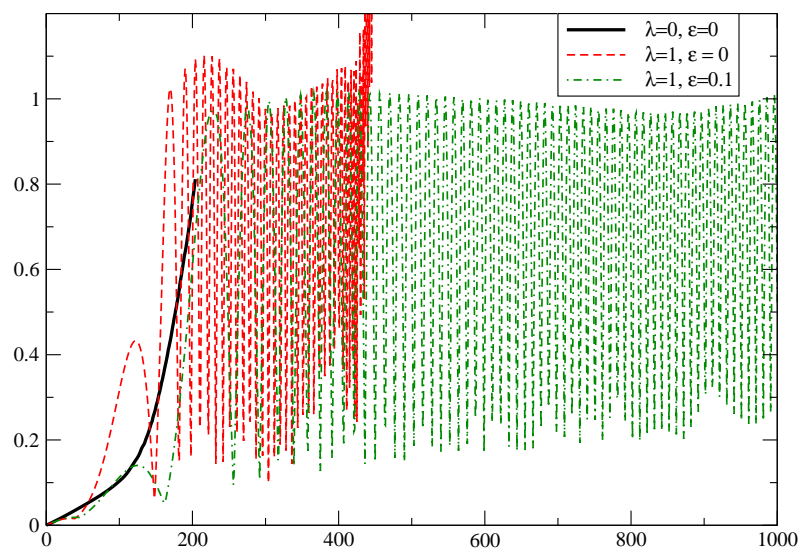

FIG. 9: The effect of constraint damping $(\lambda=1)$ and dissipation $(\epsilon=.1)$ for the $1 \mathrm{D}$ shifted gauge wave test, on an $N=100$ grid. The graph of the unscaled error $\mathcal{E}(t)$ in $g_{x x}$ shows a strong oscillation introduced by constraint damping. Although the simulation is extended by constraint damping and extended even more by dissipation, the duration of good accuracy is the same as with the bare algorithm. The constraints $\mathcal{C}^{\mu}$ exhibit similar oscillation, indicating coupling of the error to a constraint preserving mode.

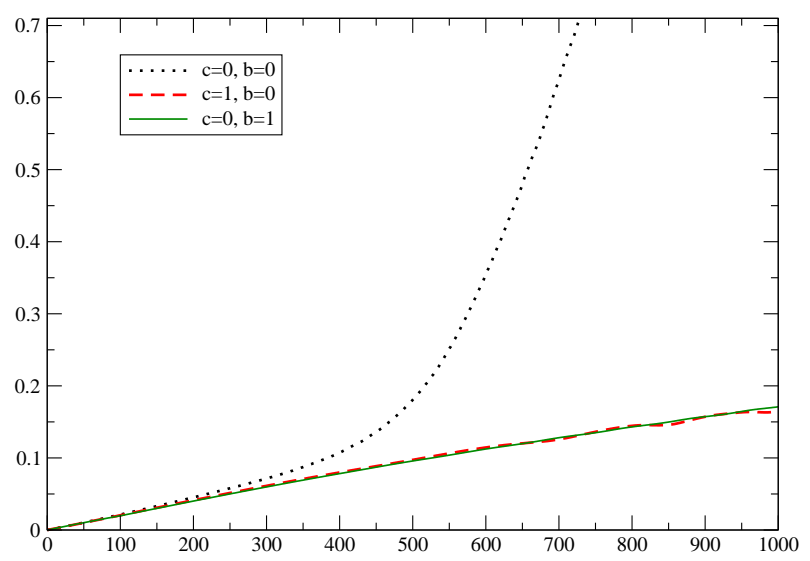

FIG. 10: Comparison plots of the unscaled error $\mathcal{E}(t)$ in $g_{x x}$ on an $N=200$ grid for the $1 \mathrm{D}$ shifted gauge wave test using the $\hat{W}$ algorithm with the constraint adjustment (4.35) with $b=1$, the constraint adjustment 4.28 with $c=1$ and the bare algorithm. The two adjustments show very similar error and both give excellent suppression of the unstable mode excited by the bare algorithm. 


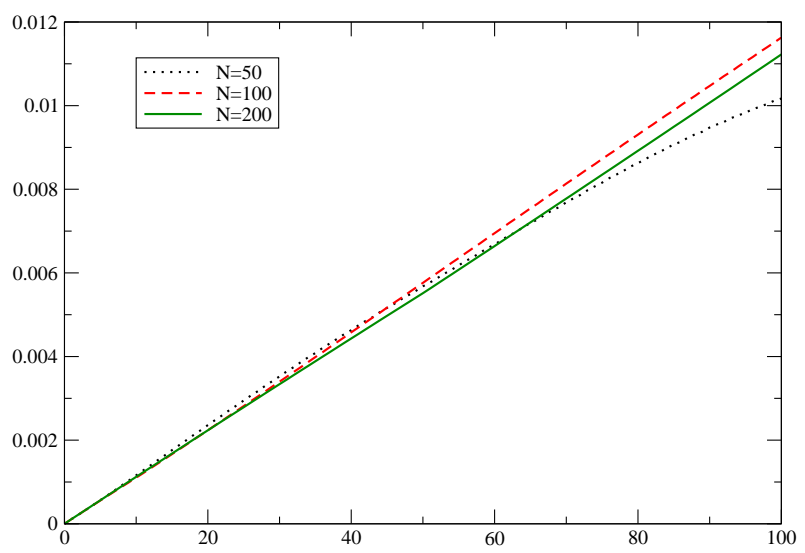

FIG. 11: Plot of the rescaled error $\mathcal{E}_{\rho}(t)$ in $g_{x x}$ for the $2 \mathrm{D}$ shifted gauge wave, with adjustment $b=1$.

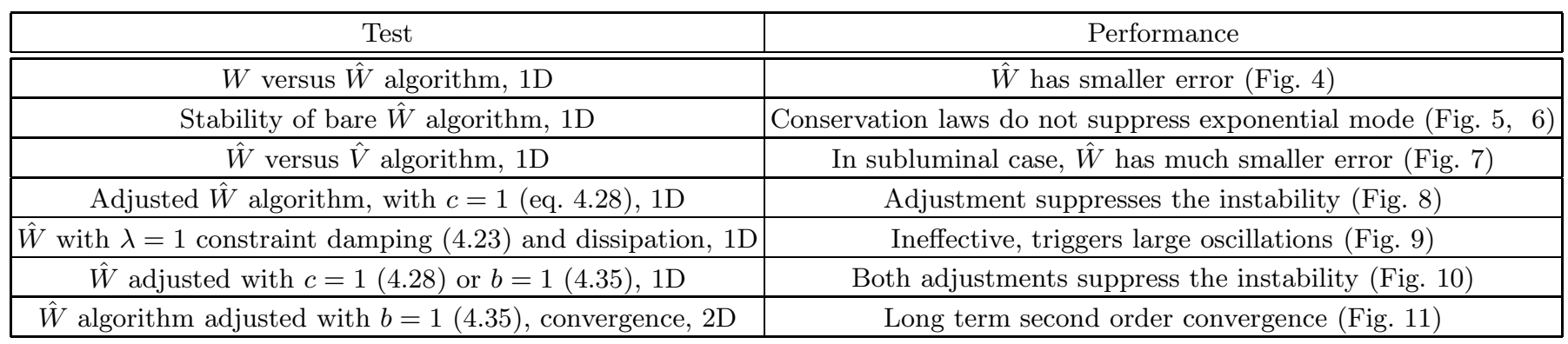

TABLE II: Summary of tests performed for the gauge wave with shift, with amplitude $A=0.5$.

difference approximations.

Our results show that discrete conservation laws for the principle part of the evolution system are a good starting point for designing an algorithm but they do not necessarily control nonlinear instabilities in the analytic problem. The same holds true for the standard harmonic reduction of the Einstein tensor. In the case of periodic boundary conditions, the techniques introduced in this paper are very effective in suppressing the long wavelength instabilities which exist in the shifted gauge wave problem. We have extended these studies of the shifted gauge wave to the initial-boundary value problem and a report is in preparation [30].

\section{Acknowledgments}

We thank H-O. Kreiss for many valuable discussions and B. Schmidt for improvements to the manuscript. This work was supported by the National Science Foundation under grant PH-0244673 to the University of Pittsburgh. We used computer time supplied by the Pittsburgh Supercomputing Center and we have benefited from the use of the Cactus Computational Toolkit (http://www.cactuscode.org).

[1] R. A. Matzner, H. E. Seidel, S. L. Shapiro, L. Smarr, W-M Suen, S. A. Teukolsky, and J. Winicour, "Geometry of a black hole collision", Science 270, 941 (1995).

[2] A. Abrahams, L. Rezzolla, M. Rupright, et al, "Schwarzschild perturbative gravitational wave extraction and outer boundary conditions", Phys. Rev. Letters 80, 1812 (1998).

[3] R. Gómez, L. Lehner, R. Marsa and J. Winicour et al, "Stable characteristic evolution of generic 3-dimensional singleblack-hole spacetimes" Phys. Rev. Letters 80, 3915 (1998).

[4] D. E. Post and R. P. Kendall, Internat. J. High Perf. Comput. Appl., 18(4), 399 (2004).

[5] D. E. Post and L. G. Votta, "Computational science demands a new paradigm", Physics Today, 58(1), 35 (2005). 
[6] The AppleswithApples Alliance, M. Alcubierre et al, "Towards standard testbeds for numerical relativity", Class. Quantum Grav., 589 (2004).

[7] www.appleswithapples.org

[8] The AppleswithApples Alliance, "Implementation of standard testbeds for numerical relativity", in preparation.

[9] H. Friedrich and A. D. Rendall, "The Cauchy problem for the Einstein equations", in Einstein's Field Equations and Their Physical Implications: Selected Essays in Honour of Jürgen Ehlers, ed. B. G. Schmidt, Springer, Berlin (2000).

[10] T. de Donder, La Gravifique Einsteinienne, (Gauthiers-Villars, Paris, 1921).

[11] V. Fock, The Theory of Space, Time and Gravitation, (MacMillan, New York, 1964).

[12] Y. Foures-Bruhat, "Theoreme d'existence pour certain systemes d'equations aux deriveés partielles nonlinaires", Acta Math 88, 141 (1952).

[13] M. C. Babiuc, B. Szilágyi and J. Winicour, "Some mathematical problems in numerical relativity", Lect. Notes Phys. (to appear) gr-qc/0404092

[14] B. Szilágyi and J. Winicour, "Well-posed initial-boundary evolution in general relativity", Phys. Rev. D68, 041501 (2003).

[15] B. Szilágyi, H-O. Kreiss and J. Winicour, "Modeling the black hole excision problem", Phys. Rev. D71, 104035 (2005).

[16] C. Bona, T. Ledvinka, C. Palenzuela and M. Zácek, "General-covariant evolution formalism for numerical relativity", Phys. Rev. D67, 104005 (2003).

[17] H. Friedrich, "Hyperbolic reductions for Einstein's equations", Class. Quant. Grav., 13, 1451 (1996).

[18] R. M. Wald, "General Relativity", University of Chicago Press (1984).

[19] A. E. Fisher and J. E. Marsden, Comm. Math. Phys., 28, 1 (1972).

[20] F. Pretorius, "Numerical Relativity Using a Generalized Harmonic Decomposition", Class.Quant. Grav. 22, 425 (2005).

[21] F. Pretorius, "Evolution of Binary Black Hole Spacetimes", Phys.Rev.Lett., 95, 121101 (2005).

[22] C. Gundlach, J. M. Martin-Garcia, G. Calabrese and I. Hinder, "Constraint damping in the Z4 formulation and harmonic gauge", Class.Quant.Grav. 22, 3767 (2005).

[23] M. Alcubierre and B. Schutz, J. Comput. Phys., 112, 44 (1994).

[24] G. Calabrese, "Finite differencing second order systems describing black hole spacetimes", Phys.Rev. D 71, 027501 (2005).

[25] G. Calabrese and C. Gundlach, "Discrete boundary treatment for the shifted wave equation", gr-qc/0509119

[26] M. Tiglio, L. Lehner and D. Neilsen, "3D simulations of Einstein's equations: symmetric hyperbolicity, live gauges and dynamic control of the constraints" Phys. Rev D70, 104018 (2004).

[27] L. Lehner, D. Neilsen, O. Reula and M. Tiglio, "The discrete energy method in numerical relativity: Towards long-term stability" Class.Quant.Grav. 215819 (2004).

[28] G. Calabrese, L. Lehner, O. Reula, O. Sarbach, M. Tiglio, "Summation by parts and dissipation for domains with excised regions", Class.Quant.Grav. 21, 5735 (2004).

[29] http://artemis.phyast.pitt.edu

[30] M. C. Babiuc, B. Szilágyi and J. Winicour, "Harmonic initial-boundary evolution in general relativity" (in preparation). 